\title{
Active Wnt signaling in response to cardiac injury
}

\author{
Martinus I. F. J. Oerlemans · Marie-José Goumans • \\ Ben van Middelaar · Hans Clevers • \\ Pieter A. Doevendans $\cdot$ Joost P. G. Sluijter
}

Received: 10 December 2009/Revised: 16 March 2010/Accepted: 23 March 2010/Published online: 7 April 2010

(C) The Author(s) 2010. This article is published with open access at Springerlink.com

\begin{abstract}
Although the contribution of Wnt signaling in infarct healing is suggested, its exact role after myocardial infarction (MI) still needs to be unraveled. We evaluated the cardiac presence of active Wnt signaling in vivo following MI, and investigated in which cell types active Wnt signaling was present by determining Axin2 promoterdriven LacZ expression. C57BL/6 Axin2-LacZ reporter mice were sacrificed at days $0,1,3,7,14$, and 21 after LAD ligation. Hearts were snap-frozen for immunohistochemistry (IHC) or enzymatically digested to obtain a single cell suspension for flow cytometric analysis. For both FACS and IHC, samples were stained for $\beta$-galactosidase and antibodies against Sca-1, CD31, ckit, and CD45.
\end{abstract}

Electronic supplementary material The online version of this article (doi:10.1007/s00395-010-0100-9) contains supplementary material, which is available to authorized users.

M. I. F. J. Oerlemans · B. van Middelaar .

P. A. Doevendans - J. P. G. Sluijter

Department of Cardiology, University Medical Centre Utrecht, Utrecht, The Netherlands

\section{M.-J. Goumans}

Department of Molecular Cell Biology,

Leiden University Medical Centre, Leiden, The Netherlands

H. Clevers

Hubrecht Institute, Utrecht, The Netherlands

P. A. Doevendans - J. P. G. Sluijter

Interuniversity Cardiology Institute of the Netherlands (ICIN),

Utrecht, The Netherlands

\section{J. P. G. Sluijter $(\bowtie)$}

Laboratory of Experimental Cardiology,

University Medical Centre Utrecht, G02.523,

PO Box 85500, 3508 GA Utrecht, The Netherlands

e-mail: J.Sluijter@umcutrecht.nl
Active Wnt signaling increased markedly in the myocardium, from 7 days post-MI onwards. Using Sca-1 and CD31, to identify progenitor and endothelial cells, a significant increase in LacZ+ cells was found at 7 and 14 days post-MI. LacZ+ cells also increased in the ckit + and CD45+ cell population. IHC revealed LacZ+ cells co-expressing Sca, CD31, CD45, vWF, and $\alpha$ SMA in the border zone and the infarcted area. Wnt signaling increased significantly after MI in Sca+- and CD31+-expressing cells, suggesting involvement of Wnt signaling in resident $\mathrm{Sca}+$ progenitor cells, as well as endothelial cells. Moreover, active Wnt signaling was present in ckit+ cells, leukocytes, and fibroblast. Given its broad role during the healing phase after cardiac injury, additional research seems warranted before a therapeutic approach on Wnt to enhance cardiac regeneration can be carried out safely.

Keywords Wnt signaling - Myocardial infarction .

Wound healing · Progenitor cell population .

Endothelial cell population

\section{Introduction}

Cardiovascular disease is one of the leading causes of morbidity and mortality in the western world [23]. Following myocardial infarction (MI), damaged myocardium is replaced with scar tissue, which may result in heart failure for which limited therapeutic options are available. One potential important signal transduction pathway involved in regulating cardiac repair and potentially stem cell maintenance and differentiation is the Wnt pathway, which plays an essential role in normal cardiac development [8]. Wnt signaling has been shown to be a key regulator of stem cell growth, differentiation, and proliferation 
in both normal homeostasis and diseased state [10, 34]. Wnt proteins form a family of highly conserved secreted signaling molecules in which the canonical $\mathrm{Wnt} / \beta$-catenin pathway is mostly studied. Upon binding of Wnt to the seven-transmembrane domain spanning frizzled (Fzd) receptor and the co-receptor lipoprotein receptor-related $5 / 6(\mathrm{Lrp} 5 / 6)$ proteins GSK3 $\beta$ is inactivated, thereby preventing the breakdown of $\beta$-catenin. After stabilization and accumulation, $\beta$-catenin enters the nucleus, where it binds to LEF/TCF transcription factors to activate the transcription of Wnt target genes.

Although extensively studied in cardiac development, the exact role of Wnt signaling after MI still needs to be unraveled. Infarct size reduction was achieved by both stimulation and inhibition of $\mathrm{Wnt} / \beta$-catenin signaling $[2,14]$, and both downregulation [4] and upregulation [32] of $\beta$-catenin gene levels were observed in cardiac hypertrophy. Moreover, epicardium-derived progenitor cells lacking $\beta$-catenin displayed impaired coronary artery formation [41], while $\beta$-catenin depletion in cardiac progenitor cells enhanced differentiation during cardiac remodeling [42]. Recent data demonstrates that $\beta$-catenin is involved in expansion of resident cardiac progenitor cells, but its role in differentiation of these cells remains controversial [20, 22, 33, 42]. Most studies investigating Wnt signaling during pathological conditions (e.g. myocardial infarction) focused on proteins participating in Wnt signal transduction such as Dishevelled-1 $[9,38]$ and $\beta$-catenin $[7,40]$. However, this not necessarily means that active $\mathrm{Wnt} / \beta$-catenin signaling, including transcription of Wnt target genes, is present.

Considering its potency to act as a therapeutic target, fundamental insights on Wnt time-dependency and cell specificity upon injury in the adult injured myocardium are necessary. Therefore, we evaluated the presence of active Wnt signaling in vivo in the heart following MI. Moreover, we investigated in which cell types active Wnt signaling was present during different phases following MI.

After binding of $\beta$-catenin to LEF/TCF transcription factors in the nucleus, several Wnt target genes are activated, including the Axin2 gen [21]. Axin2 is able to downregulate $\beta$-catenin and acts as a negative regulator of Wnt signaling [16]. We used Axin $2^{+/ L a c Z}$ reporter mice in which the LacZ gene is under the control of the Axin2 promoter, providing a reliable way to detect Wnt activity by visualizing LacZ-reporter-positive cells [24].

\section{Materials and methods}

\section{Animals}

Male and female C57BL/6 Axin2 ${ }^{+/ L a c Z}$ reporter mice were bred and used at $8-10$ weeks of age. For generation of the
Axin2-lacZ mouse [24], the $\beta$-galactosidase (NLS-lacZ) gene was introduced in frame to the endogenous Axin2 promoter by homologous recombination, thereby replacing most of exon 2 (MGI Ref ID J:74286) but leaving the Axin2 promoter intact. All experiments were approved by the Animal Experimentation Committee of the Utrecht University and were in accordance with the Guide for the Care and Use of Laboratory Animals of the Institute of Laboratory Animal Resources. The investigation conforms to the Guide for the Care and Use of Laboratory Animals published by the US National Institutes of Health (NIH Publication No. 85-23, revised 1996).

\section{Myocardial infarction}

The MI was induced by ligation of the left anterior descending (LAD) coronary artery under isoflurane anesthesia, as described previously [37, 39]. Briefly, mice were anesthetized with isoflurane, orally intubated, and ventilated. A left thoracotomy was performed at the third intercostal space, and muscles and pericardium were dissected. LAD ligation was performed with an 8-0 nonabsorbable ethilon suture. After verification that coronary occlusion had occurred by the change of color and kinesis of the apex and anterior-lateral wall, the thorax was closed in layers. After detubation, mice were kept warm until fully recovered. Mice were sacrificed at baseline ( 0 day, controls) and at 1, 3, 7, 14, and 21 days after MI. The hearts were flushed with phosphate-buffered saline (PBS) and dissected. The left ventricle was cut in two halves through the center of the infarct along the longitudinal axis. One half was snap-frozen in liquid nitrogen and stored at $-80^{\circ} \mathrm{C}$; the other half was kept in PBS for further processing. Remote area was defined as the non-infarcted part of the interventricular septum.

\section{Cell isolation and flow cytometry analysis}

Freshly dissected hearts, containing the infarcted and noninfarcted area, were perfused with PBS and washed, minced into $1-2 \mathrm{~mm}^{2}$ pieces, digested for $45 \mathrm{~min}$ at $37^{\circ} \mathrm{C}$ with $10 \mathrm{mg} / \mathrm{ml}$ collagenase A (Roche), and passed through a $70-\mu \mathrm{m}$ filter. Remaining cells were plated on a 12-well plate DMEM with $10 \%$ fetal bovine serum (FBS) as described previously [36] or aliquoted after centrifugation for flow cytometric analysis. After centrifugation, cells were resuspended in PBS containing 4\% FBS, and aliquots containing $1.0 \times 10^{6}$ cells were stained. Single cell suspensions were stained with FDG (Fluoreporter ${ }^{\circledR}$ LacZ Kit F-1930, Molecular Probes) to detect $\beta$-galactosidase activity. Next, isolated cells were labeled with antibodies against Sca-1 (BD Pharmingen 553108, PE conjugated), isotype control (BD Pharmingen, 553930), CD31 
(Biolegend 102417, Pe-Cy7 conjugated), isotype control (Biolegend, 400521), ckit (Abcam 46790, APC-Cy5.5 conjugated), isotype control (Abcam 46745), and CD45 (Abcam 51482, PE-Texas Red conjugated). To exclude dead cells from analysis (including cardiomyocytes), 7-AAD (BD 559925) was used. Samples were analyzed by flow cytometry (Beckman Coulter Cytomics FC500 FACS), collecting 15,000-50,000 events per sample.

Histology and immunohistochemistry/ immunocytochemistry

Frozen sections of 5-7 $\mu \mathrm{m}$ were cut in a cryostat (Microm HM560, Cryo-Star) and mounted on silane-BSA-coated slides. Histologic analysis of MI was performed by $\mathrm{H} \& \mathrm{E}$ staining. Sections were fixed in acetone, blocked with $10 \%$ normal goat serum (NGS), and incubated overnight with primary antibody. After incubation with secondary antibody for $1 \mathrm{~h}$, sections were washed in PBS and mounted in Fluoromount (Southern Biotech). For immunocytochemistry, coverslips with cultured cells were fixed in $4 \%$ paraformaldehyde at room temperature and permeabilized with $0.2 \%$ Triton X-100 in PBS. Cells were blocked with $2 \%$ bovine serum albumin (BSA) for $15 \mathrm{~min}$ and incubated overnight at $4{ }^{\circ} \mathrm{C}$ with primary antibody with $10 \%$ NGS. The coverslips were then incubated with secondary antibody in PBS with $10 \%$ NGS for $1 \mathrm{~h}$ and subsequently mounted. For both IHC and ICC, Hoechst dye was used to visualize nuclei. Primary antibodies for Sca-1 (BD Pharmingen, 57403), CD31 (BD Pharmingen, 550274), CD45 (BD Pharmingen 553774), tropomyosin (Sigma, T9283), $\beta$-galactosidase (Abcam ab616), and von Willebrand Factor (Dako, M0616) were used with matching isotypes and a FITC-conjugated antibody against alpha-smooth muscle actin (Sigma, A2547). Secondary antibodies were conjugated with AF555 (Invitrogen A21429), AF488 (Invitrogen A11001), and AF647 (Invitrogen A21247). Slides and coverslips were examined with a Nikon light microscope equipped for epifluorescence or Zeiss LSM 510 Meta confocal microscope for high magnification capturing. All images illustrated are representative of least three independent experiments.

\section{$\beta$-Galactosidase (LacZ) staining on cultured cells}

Cells were fixed in $0.2 \%$ paraformaldehyde for $10 \mathrm{~min}$, followed by a 10 -min wash in PBS with $2 \mathrm{mM} \mathrm{MgCl}_{2}$ on ice. Cells were then placed in Xgal staining solution $(2 \mathrm{mM}$ $\mathrm{MgCl}_{2}, 0.1 \%$ sodium deoxycholate and $0.02 \% \mathrm{NP}-40$, $5 \mathrm{mM} \mathrm{K}_{3} \mathrm{FE}(\mathrm{CN})_{6}, 5 \mathrm{mM} \mathrm{K}_{4} \mathrm{Fe}(\mathrm{CN})_{6}$, and $1 \mathrm{mg} / \mathrm{ml} \mathrm{X}$-gal (Invitrogen) in $\mathrm{PBS}$ ) at $37^{\circ} \mathrm{C}$ for $30 \mathrm{~min}$. Thereafter, cells were washed twice in PBS with $2 \mathrm{mM} \mathrm{MgCl}_{2}$ at room temperature and covered with coverslips and examined by light microscopy.

\section{Statistics}

Data (mean \pm SEM) were analyzed by Mann-Whitney $U$ test, using a significance level of $P<0.05$ (SPSS for Windows, v15.0).

\section{Results}

Increased number of cells displays active Wnt signaling in the heart after myocardial infarction

To identify Wnt signaling in the adult mouse heart, we used the Axin2-LacZ reporter mouse which will result in LacZ expression in those cells that have active canonical Wnt signaling. To demonstrate specificity of $\beta$-galactosidase activity only in cells having active Wnt signaling, a single cell suspension was made from Axin2 reporter hearts and put into culture. Immunocytochemistry revealed the presence of LacZ-expressing cells (and thus canonical Wnt signaling) in the culture, while cell cultures from wild type hearts did not show any positive staining (Fig. 1). Moreover, Wnt-reporter-positive staining increased upon stimulation with lithium chloride, a strong chemical inducer of Wnt signaling (Supplementary Fig. 1). In order to quantify the number of Wnt-reporter-expressing cells (LacZ+ cells), we fluorescently labeled single cell suspensions with FDG. Flow cytometry analysis revealed that in the heart of adult Axin2-reporter mice, a LacZ-expressing cell population of $9.55 \pm 0.78 \%$ is present (Fig. 2a), while no $\beta$-galactosidase activity could be observed in non-transgenic hearts (Fig. 2a, right panel). Next, we analyzed whether Wnt signaling increased following myocardial infarction (MI). At 7 days post-MI, the number of LacZ+ cells had increased significantly (14.47 $\pm 0.75 \%)$ compared to basal levels of non-operated mice (Fig. 2b). Since the number/percentage of LacZ+ cells at day 3 post-MI was still comparable with basal levels $(9.44 \pm 0.30 \%)$, the increase in active Wnt signaling started between days 3 and 7 post-MI. LacZ+ cells increased further on 14 days $(22.42 \pm 0.97 \%)$ and 21 days $(24.98 \pm 1.37 \%)$. Although there was a significant increase in LacZ+ cells between days 7 and 14, the number of LacZ-expressing cells stabilized between days 14 and 21 post-MI. Quantitative RT-PCR on temporal Axin2 expression confirmed the Axin2 promoter-driven $\beta$-galactosidase activity after myocardial infarction.

LacZ-expressing cells increase significantly throughout the whole myocardium

To localize active Wnt signaling in the injured heart, we analyzed $\beta$-galactosidase expression by staining cardiac 
Fig. 1 LacZ staining on cultured cells. Cells were isolated as was done for flow cytometric analysis.

Axin $2^{+/ L a c Z}$ and wild-type

(WT) isolated cells were cultured in DMEM with $10 \%$ fetal bovine serum (FBS) and fluorescently stained for $\beta$-galactosidase (red) and Hoechst (nuclei, blue) to detect the active Wnt signaling. a, c LacZ-expressing cells (and thus canonical Wnt signaling) are present in the culture, while cell cultures from wild-type hearts did not show any positive staining (b, d). Top

$\beta$-galactosidase staining, bottom $\beta$-galactosidase staining merged with Hoechst nuclear staining.

Scale bar $20 \mu \mathrm{m}, 400 \times$

magnification

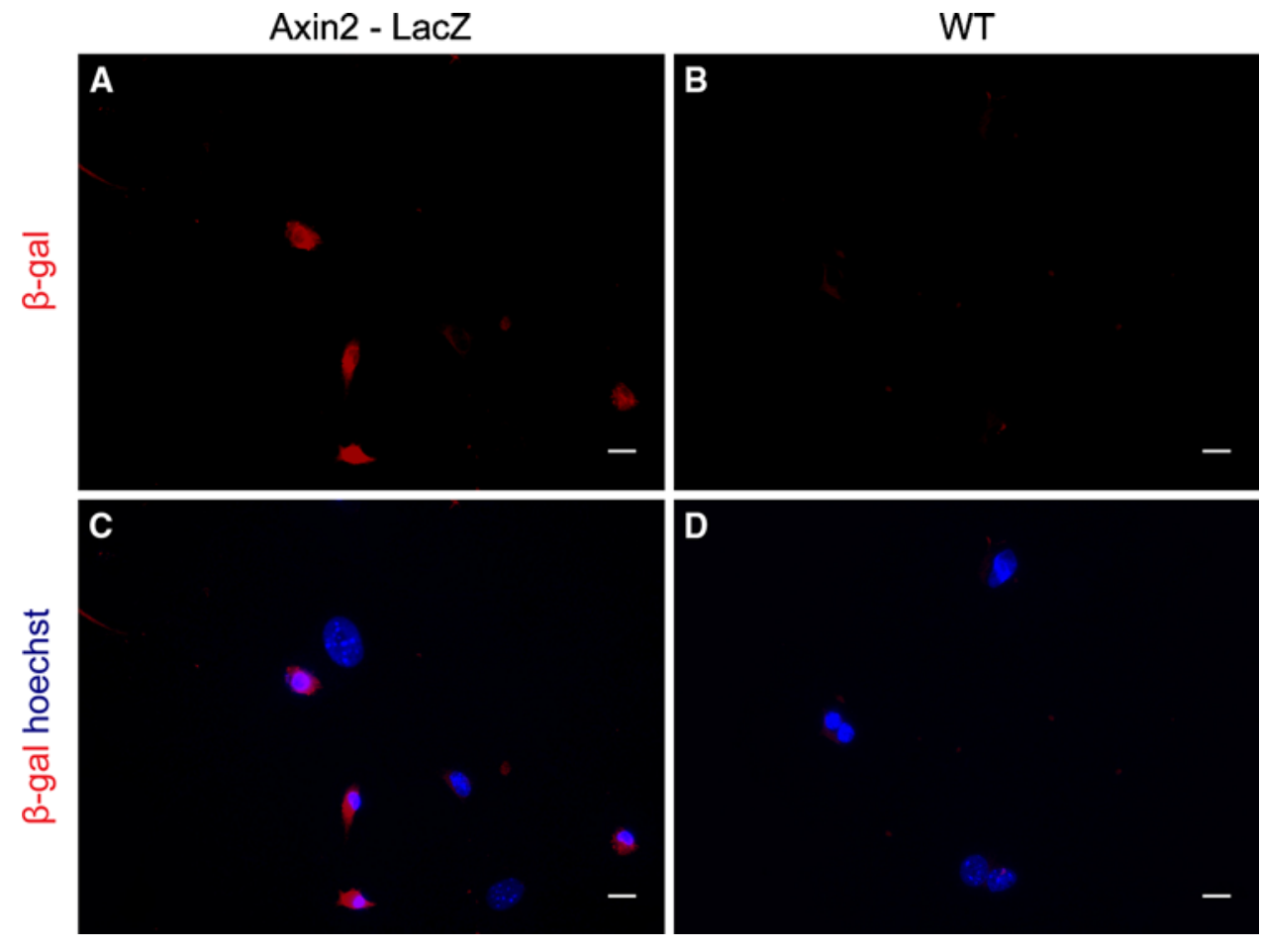

A LacZ reporter mouse
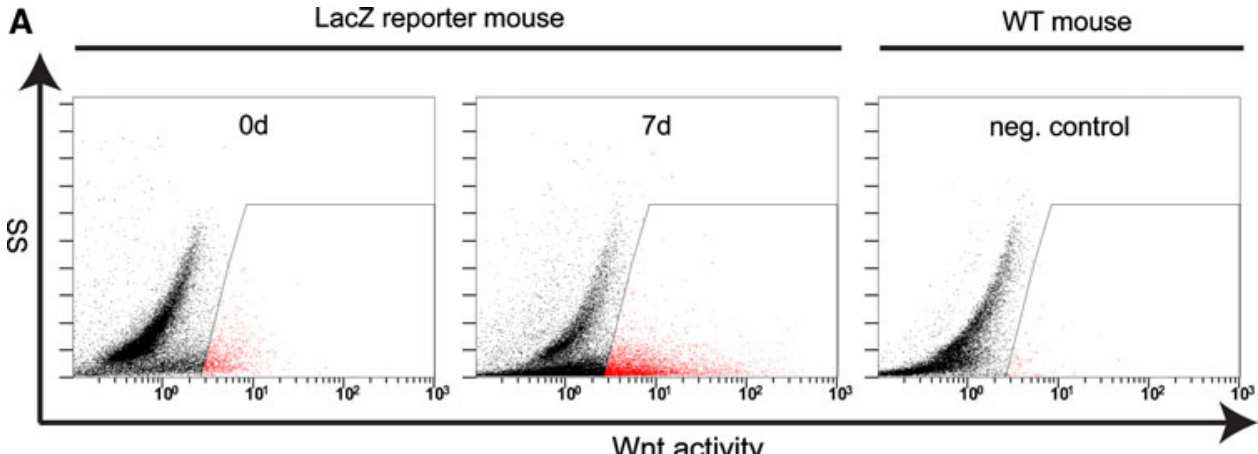

Wnt activity

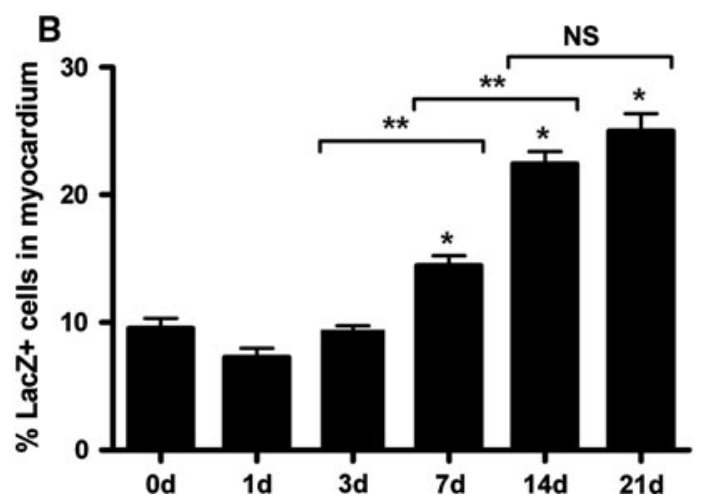

Fig. 2 Increased Wnt signaling after myocardial infarction. Freshly dissected hearts were digested into a single cell suspension and stained with FDG to detect $\beta$-galactosidase activity. a Representative flow cytometric scatter plots of a control heart (0 day, left window) together with a sample (7 days, middle window) of the LacZ-reporter mouse after myocardial infarction (MI). Wnt+ cell population increased in response to MI, while wild-type samples showed no

Wnt activity (right window). b Flow cytometric analysis showed a significant increase in LacZ+ cells on days 7, 14, and 21 after MI compared to baseline levels of a non-operated mouse. A significant difference in the LacZ+ cells was observed between days 3-7 and days 7-14. After 14 days, the amount of Wnt+ cells started to stabilize. $* P<0.01$ compared to control, $* * P<0.01$ between groups, $n=5-10$ per group 
Fig. $3 \mathrm{LacZ}+$ cells increase significantly throughout the whole myocardium. Frozen sections of mouse hearts from different time points after MI were fluorescently stained for $\beta$-galactosidase (red) and Hoechst (nuclei, blue) to localize LacZ+ cells.

a Overview of a mouse heart 7 days after infarction stained for tropomyosin (green). BZ border zone; $M I$ myocardial infarction $(12 \times$ magnification, scale bar $0.5 \mathrm{~mm})$.

b Representative section of a control heart showing some LacZ+ nuclei (purple spots). c Representative section of a mouse heart 14 days after MI, showing an increase in LacZ+ nuclei (purple), as well as more active Wnt signaling in the surrounding tissue in the border zone. Scale bar $20 \mu \mathrm{m}$,

$630 \times$ magnification.

d, e Quantification of LacZ+ cells based on LacZ+-positive nuclei compared to total nuclei in border zone and remote area The number of LacZ+ cells increased significantly after MI in both the remote area and the border zone. $* P<0.05$ compared to control, $* * P<0.05$ between groups, $n=6-8$ per group


sections from different time points post-MI. In all sections stained, we observed LacZ-positive cells throughout the whole myocardium, including border zone and remote area. LacZ+ cells were also found in the infarcted area although not as much as in the border zone. The number of LacZ+ nuclei increased compared to control sections (Fig. 3a-c). LacZ + nuclei were counted and compared with the total number of nuclei in both the border zone and the remote area to see whether the increase of LacZ+ cells was restricted to a particular area. Active Wnt signaling increased significantly in both the remote area and the border zone (Fig. 3d, e). The increase in LacZ+ cells showed a peak around 14 days post-MI and started to normalize between 14 and 21 days. Although to a large extent upregulated in the border zone, active Wnt reporter cells can be found throughout the whole myocardium, showing a significant increase in response to myocardial injury.

LacZ + cells increase significantly in the progenitor, endothelial, and leukocyte cell population

To characterize the composition of the LacZ+-positive cell population in the myocardium, we used Sca-1 and ckit (CD117) to identify progenitor populations, CD31 to detect endothelial cells; CD45 was used as a common leukocyte marker, using flow cytometry. LacZ+ cells displayed expression of both Sca-1 and CD31 on their cell surface (Fig. 4a). Active Wnt-reporter cells were also found to express ckit and CD45, the latter only at 3 and 7 days post-MI. Immuno labeling on different sections after MI confirmed the flow cytometric analysis, demonstrating 
Fig. $4 \mathrm{LacZ}+$ cells are present in the progenitor, endothelial, and leukocyte cell population. a Characterization of LacZ+ cells by co-expression of Sca-1, CD31, ckit, and CD45 using flow cytometry. Solid histograms represent isotype control antibodies. All markers studied were present within the LacZ+ cell population. Using immunohistochemistry to reveal co-localization, frozen sections were fluorescently stained for $\beta$-galactosidase (red), Hoechst (nuclei, blue), tropomyosin (green), Sca-1 (b), CD31 (c), and CD45 (d). Confocal microscopy showed that LacZ+ cells co-localize with progenitor, endothelial and leukocyte cell populations (red arrows), confirming flow cytometric analysis. Scale bar $20 \mu \mathrm{m}, 630 \times$ magnification


co-localization of LacZ+ nuclei with Sca-1, CD31, and CD45 (Fig. 4b-d). Since CD31 can be expressed on nonendothelial cells as well, we confirmed that LacZ+ nuclei were present in vessels in the border zone by using von Willebrand factor (vWF) (Supplementary Fig. 2a). Furthermore, using alpha-smooth muscle actin ( $\alpha$ SMA), we found fibroblast (Supplementary Fig. 2b) and vascular smooth muscle cells (Supplementary Fig. 2c) with LacZ+ nuclei in the border zone and infarct, respectively. Cytoplasmic LacZ-positive staining was also observed mainly in the infarcted area, where clusters of cardiomyocytes were still present. This is probably caused by either nuclear leakage from dying cardiomyocytes or senescence-associated $\beta$-galactosidase activity [25]. Therefore, not only cardiac progenitor cells and endothelial cells demonstrated LacZ positivity but also leukocytes, fibroblasts, and myocytes.

Subsequently, we analyzed whether changes in the active Wnt signaling was present in specific cell populations, exploring the role of Wnt signaling in different cell types that play an important role after MI. Flow cytometric analysis indeed displayed a significant increase of LacZ + cells in both Sca-1+ and CD31+ cell populations, parallel with an increase in total Sca + and CD31+ cell numbers (Fig. 5a-d).
The total number of Sca+ cells increased significantly between 3 and 7 days post-MI, but started to decrease again after 14 days (Fig. 5a). A similar pattern was found for CD31+ cells although the number of cells at 7, 14, and 21 days remained significantly higher, compared to baseline levels (Fig. 5c). LacZ+ cells increased significantly in Sca+ and CD31+ cell populations, starting from day 7 post-MI. Ckit + cells also increased significantly after MI, showing a peak at 14 days post-MI. This increase started between days 7 and 14, still having higher levels at 21 days, when compared to baseline (Fig. 5e). LacZ+ cells in the ckit+ population increased gradually after MI with highest level at 3 days (Fig. 5f). The CD45+ cell population increased after MI, showing a significant increase at 3, 7, and 14 days postMI, followed by a gradual decrease toward basal levels at 21 days (Fig. 5g). Interestingly, the number of LacZ+ cells in CD45+ cell population increased significantly as well, following almost the same trend as the total CD45+ population with peaks at 3 and 7 days post-MI (Fig. 5h).

Additionally, we tested whether different subsets of the LacZ-positive cells changed during time following MI. Since it has been suggested that cardiac progenitor cells express Sca-1, we tested LacZ expression in Sca+/CD31cells. Wnt signaling increased significantly in $\mathrm{Sca}+/$ 
Fig. $5 \mathrm{LacZ}+$ cells increase significantly in the progenitor, endothelial, and leukocyte cell population. Flow cytometric analysis showing a significant increase of LacZ + cells in both Sca-1+ (b) and CD31+ (d) cell populations, parallel with an increase in total Sca+ (a) and CD31+ (c) cell numbers. Total ckit $+(\mathbf{e})$ and $\mathrm{CD} 45+(\mathbf{g})$ cell populations increased significantly as well. LacZ+ cells in ckit + cell population increased with a peak at day 3 post-MI (f). Within the CD45+ leukocyte cell population, LacZ + cells showed a significant increase at days 3,7 , and 14 days post-MI (h). ${ }^{*} P<0.05$ compared to control, $* * P<0.05$ between groups, a-d $n=5-10$ per group, e-h $n=3-5$ per group


CD31 - cell population, showing a peak at 7 days after MI (Fig. 6a). The endothelial cell population (Sca-/CD31+ cells) displayed a significant increase in Wnt signaling at 7 and 14 days after MI (Fig. 6b). Furthermore when looking at the Sca-/CD31 - cell population, probably representing inflammatory (e.g. CD45+) and other circulating cell populations, an increase was found at 3 days post-MI (Fig. 6c). At all studied time points, Sca+/CD31+ cell population formed the largest population of LacZ + cells (Fig. 6d). However, this subpopulation decreased significantly starting from day 3 , reaching the lowest levels at 7 days post-MI (Fig. 5d). When looking at the changes in 



Fig. 6 Sca+/CD31- and Sca-/CD31+ cells increase significantly in LacZ + cell population. Flow cytometric analysis of specific subsets of LacZ+ cells, based on the co-expression of Sca-1 and $\mathrm{CD} 31$ to distinguish between progenitor $(\mathrm{Sca}+/ \mathrm{CD} 31-)$ and endothelial $(\mathrm{Sca}-/ \mathrm{CD} 31+)$ cell populations. a The amount of Sca+l CD31 - progenitor cells increased significantly within the LacZ+ cell population 7 days post-MI. b The amount of Sca-/CD31+ endothelial cells increased significantly within the LacZ + cell population after infarction. c The amount of Sca-/CD31- progenitor cells increased significantly within the LacZ + cell population 3 days post-

MI. d Sca+/CD31+ cell population formed the largest population of LacZ + cells, but decreased significantly starting from 3 days after infarction. $* P<0.05$ compared to control, $* * P<0.05$ between groups, $n=5-10$ per group. e Cumulative overview of changes in Wnt + cell population during time, based on the co-expression of Sca1 and CD31. Sca+/CD31+ cells, although decreasing in time, formed the majority within the LacZ+ cell population. An early increase in the Sca-/CD31- cell fraction, probably CD45+ cells, can be appreciated. Both Sca+/CD31- and Sca-/CD31+ cell fractions increased significantly starting at day 7 after MI

cells showing active Wnt signaling in time, more than $50 \%$ of the LacZ+ cells were either Sca+/CD31+ or Sca-/ CD31 - at all time points (Fig. 6e). Although a significant increase in Sca+/CD31- progenitor cells and Sca-/ $\mathrm{CD} 31+$ endothelial cells was observed, these two subsets of cells together did not exceed $40 \%$ of the LacZ+ cell population.

\section{Discussion}

Wnt signaling is an important regulatory pathway in biology and behavior of stem cells [10,34], and is required for normal cardiac development $[8,11]$. In response to cardiac stress and injury, reactivation of $\mathrm{Wnt} / \beta$-catenin signaling takes place, probably being part of a fetal gene 
reprogramming required for tissue repair [18, 30]. Considering its potency to be used as a therapeutic target, it is of great interest to gain more information on the function of Wnt signaling in the adult heart, especially in the context of cardiac tissue repair. However, during the last few years, contradictory results were reported upon inhibition and stimulation of Wnt with respect to the adaptive response on hypertrophic and ischemic stimuli $[2-4,14,38]$. It is becoming clear that Wnt molecules do not simply drive proliferation or differentiation, but rather regulate and finetune these processes in a cell-type and time-dependant manner. There are $19 \mathrm{Wnt}$ proteins, 10 Fzd receptors, and 2 Lrp co-receptors in mammals, representing a complex regulatory family.

In our study, cell-specific Wnt signaling and its localization was visualized in response to cardiac injury using Axin $2^{+/ L a c Z}$ reporter mice. Although an indirect measurement of the active Wnt cascade, the not cell-specific role of Axin2 in the Wnt response [10] makes it a reliable way to detect the canonical Wnt activity. We demonstrate that active Wnt signaling is significantly upregulated in those cell populations that are considered to play a role in cardiac wound healing after MI and in cardiomyocytes. Active Wnt signaling was significantly increased in the border zone and remote area after cardiac injury. Together with an increase in total progenitor, endothelial, and leukocyte cell populations, the number of LacZ + cells in these populations increased significantly. In addition, a significant increase in $\mathrm{Sca}+/ \mathrm{CD} 31-$ progenitor and Sca-/CD31+ endothelial cell number was found within the population that showed active Wnt signaling. Moreover, we observed active Wnt signaling in fibroblasts, vWF-positive endothelial cells, vascular smooth muscle cells $(\alpha \mathrm{SMA}+)$, fibroblasts $(\alpha \mathrm{SMA}+)$, and cytoplasm of cardiomyocytes.

Whether we can enhance the cardiac repair by modulating Wnt remains the question since active Wnt signaling was present in all the cell populations studied. Different compounds are available already that can interfere with Wnt signaling, but up to now Wnt modulation after myocardial infarction did not result in consistent results. Frizzled or secreted frizzled-related proteins (sFRPs) can compete for Wnt binding, thereby antagonizing Wnt signaling. Transgenic mice overexpressing Frizzled-A, a member of the frizzled family, displayed less apoptosis and a higher capillary density resulting in reduced infarct size as compared to wild-type animals [2]. Others reported that mesenchymal stem cells (MSCs) overexpressing sFRPs showed better survival after injection into the peri-infarct region. This resulted in increased engraftment and vascularized granulation tissue after MI [1, 27], probably by antagonizing Wnt3a which resulted in less apoptosis [43]. On the contrary, direct $\beta$-catenin injection in the border zone of rats after coronary artery ligation decreased infarct size and promoted cell survival in both cardiomyocytes and cardiac fibroblasts [14]. Furthermore, cardioprotection by ischemic preconditioning was shown after GSK3 $\beta$ inhibition [17], due to induction of neovascularization and inhibition of apoptosis. The opposite effect was demonstrated by sFRP1 overexpression [3]. Recently, fibrosis was shown to be limited in SFRP1 null mice after myocardial infarction [19] although regulated via mechanisms independently of Wnt signaling. As indicated, different Wntdependent and -independent stimuli seem to interact with GSK $3 \beta$ and sFRPs with respect to cell survival and repair $[15,28]$.

In our study, Sca+ progenitor, as well as endothelial cells, and cardiomyocytes showed an increased LacZ expression, and these LacZ+ cells increased in number. This increase in number suggests that increased Wnt signaling might influence survival or proliferation of these cells in response to cardiac injury. Furthermore, ckit+ and CD45+ cell populations showed increased LacZ expression as well. Although this association was observed, our study is purely descriptive and does not allow to conclude any causal relationship between an increased presence of LacZ and the different cell numbers. Interestingly, studies aiming at wound healing after MI reported an upregulation of $\beta$-catenin in vascular endothelial cells during neovascularization [7, 9] and in proliferating and migrating myofibroblasts [9] after MI. Consistent with these studies, our findings indirectly suggest that Wnt signaling drives expansion of different cell types, including Sca+/CD31- progenitor and Sca-/CD31+ endothelial cells, as well as in ckit + and CD45+ cell populations, in response to cardiac injury. The upregulation of $\beta$-catenin observed in these studies does not necessarily mean that a higher promoter activity is present though. Whether increased Wnt signaling directly influences cardiac wound healing or cell survival remains unanswered.

A second question is whether Wnt signaling modulates differentiation after cardiac injury. Several studies reported that canonical and non-canonical Wnt signaling are required for the differentiation of cardiac progenitor cells [20], and enhances the cardiomyogenic potential of bone marrow cells [13]. Different progenitor cell populations, resident in the adult heart, have been shown to differentiate into cardiomyocytes or vascular structures both in vitro and in vivo $[5,6,29]$. Although stimulation of endogenous regeneration capacity might offer a new treatment modality, activation of the resident cardiac progenitor cells to repair the injured myocardium still remains a matter of debate. From this point, it is at least striking that we observed a significant increase in Sca+/CD31- progenitor cells and $\mathrm{Sca}-/ \mathrm{CD} 31+$ endothelial cells within the LacZ+ population. LacZ+ cells expressing both $\mathrm{Sca}+$ and CD31+ 
decreased after MI. These shifts within the LacZ+ populations might suggest the involvement of Wnt signaling in the process of differentiation in response to MI. Several other reports have been published supporting a role for Wnt signaling in the expansion and proliferation of cardiac progenitor cells during development $[11,12,22]$. Canonical and non-canonical Wnt signaling has been shown to stimulate the cell growth and survival in isolated endothelial cells in vitro $[26,35,40]$. Moreover, $\beta$-catenin stabilization via lithium stimulation and administration of different Wnt molecules induced muscle regeneration via differentiation of Sca+/CD45+ cells, which was reduced upon sFRP2/3 injection [31]. In contrast, cardiac-specific $\beta$-catenin depletion was shown to attenuate cardiac remodeling, mainly through enhanced differentiation of $\mathrm{Sca}+$ progenitor cells [42].

In conclusion, the present study demonstrates a temporal upregulation of the active Wnt signaling after MI which is not restricted to a particular area of the heart. LacZ+ cells were shown to co-express progenitor, endothelial, leukocyte, and fibroblast markers, suggesting a broad role of Wnt in reaction to cardiac injury. Interestingly, different cell populations displayed a distinct LacZ response in time, suggesting a time- and cell-specific activation of Wnt after MI. When considering Wnt reactivation as a therapeutic approach to enhance cardiac regeneration, one should consider this time- and cell-specific expression window. Based on the significant upregulation of the progenitor and endothelial cell populations that show the active Wnt signaling, it is possible that Wnt modulates cardiac regeneration and neovascularization during the healing phase after MI. However, as more populations are involved, this also illustrates its complexity.

Acknowledgments This work was supported by a VIDI grant (016.056.319) from the Netherlands Organization for Scientific Research (NWO), the Netherlands Heart Foundation (2003B07304), BSIK program "Dutch Program for Tissue Engineering", grants 6746, Bekalis price (PD) and "Stichting Swaeneborgh" (MO).

Open Access This article is distributed under the terms of the Creative Commons Attribution Noncommercial License which permits any noncommercial use, distribution, and reproduction in any medium, provided the original author(s) and source are credited.

\section{References}

1. Alfaro MP, Pagni M, Vincent A, Atkinson J, Hill MF, Cates J et al (2008) The Wnt modulator sFRP2 enhances mesenchymal stem cell engraftment, granulation tissue formation and myocardial repair. Proc Natl Acad Sci USA 105:18366-18371

2. Barandon L, Couffinhal T, Ezan J, Dufourcq P, Costet P, Alzieu P et al (2003) Reduction of infarct size and prevention of cardiac rupture in transgenic mice overexpressing FrzA. Circulation $108: 2282-2289$
3. Barandon L, Dufourcq P, Costet P, Moreau C, Allieres C, Daret D et al (2005) Involvement of FrzA/sFRP-1 and the Wnt/frizzled pathway in ischemic preconditioning. Circ Res 96:12991306

4. Baurand A, Zelarayan L, Betney R, Gehrke C, Dunger S, Noack $C$ et al (2007) Beta-catenin downregulation is required for adaptive cardiac remodeling. Circ Res 100:1353-1362

5. Bearzi C, Rota M, Hosoda T, Tillmanns J, Nascimbene A, De Angelis A et al (2007) Human cardiac stem cells. Proc Natl Acad Sci USA 104:14068-14073

6. Beltrami AP, Barlucchi L, Torella D, Baker M, Limana F, Chimenti S et al (2003) Adult cardiac stem cells are multipotent and support myocardial regeneration. Cell 114:763-776

7. Blankesteijn WM, van Gijn ME, Essers-Janssen YP, Daemen MJ, Smits JF (2000) Beta-catenin, an inducer of uncontrolled cell proliferation and migration in malignancies, is localized in the cytoplasm of vascular endothelium during neovascularization after myocardial infarction. Am J Pathol 157:877-883

8. Brade T, Manner J, Kuhl M (2006) The role of Wnt signalling in cardiac development and tissue remodelling in the mature heart. Cardiovasc Res 72:198-209

9. Chen L, Wu Q, Guo F, Xia B, Zuo J (2004) Expression of Dishevelled-1 in wound healing after acute myocardial infarction: possible involvement in myofibroblast proliferation and migration. J Cell Mol Med 8:257-264

10. Clevers H (2006) Wnt/beta-catenin signaling in development and disease. Cell 127:469-480

11. Cohen ED, Tian Y, Morrisey EE (2008) Wnt signaling: an essential regulator of cardiovascular differentiation, morphogenesis and progenitor self-renewal. Development 135:789798

12. Cohen ED, Wang Z, Lepore JJ, Lu MM, Taketo MM, Epstein DJ et al (2007) Wnt/beta-catenin signaling promotes expansion of Isl-1-positive cardiac progenitor cells through regulation of FGF signaling. J Clin Invest 117:1794-1804

13. Flaherty MP, Abdel-Latif A, Li Q, Hunt G, Ranjan S, Ou Q et al (2008) Noncanonical Wnt11 signaling is sufficient to induce cardiomyogenic differentiation in unfractionated bone marrow mononuclear cells. Circulation 117:2241-2252

14. Hahn JY, Cho HJ, Bae JW, Yuk HS, Kim KI, Park KW et al (2006) Beta-catenin overexpression reduces myocardial infarct size through differential effects on cardiomyocytes and cardiac fibroblasts. J Biol Chem 281:30979-30989

15. Heusch G, Boengler K, Schulz R (2010) Inhibition of mitochondrial permeability transition pore opening: the holy grail of cardioprotection. Basic Res Cardiol 105:151-154

16. Jho EH, Zhang T, Domon C, Joo CK, Freund JN, Costantini F (2002) Wnt/beta-catenin/Tcf signaling induces the transcription of Axin2, a negative regulator of the signaling pathway. Mol Cell Biol 22:1172-1183

17. Kaga S, Zhan L, Altaf E, Maulik N (2006) Glycogen synthase kinase-3beta/beta-catenin promotes angiogenic and anti-apoptotic signaling through the induction of VEGF, Bcl-2 and survivin expression in rat ischemic preconditioned myocardium. J Mol Cell Cardiol 40:138-147

18. Kim Y, Phan D, van RE, Wang DZ, McAnally J, Qi X et al (2008) The MEF2D transcription factor mediates stress-dependent cardiac remodeling in mice. J Clin Invest 118:124-132

19. Kobayashi K, Luo M, Zhang Y, Wilkes DC, Ge G, Grieskamp T et al (2009) Secreted Frizzled-related protein 2 is a procollagen C proteinase enhancer with a role in fibrosis associated with myocardial infarction. Nat Cell Biol 11:46-55

20. Kwon C, Arnold J, Hsiao EC, Taketo MM, Conklin BR, Srivastava D (2007) Canonical Wnt signaling is a positive regulator of mammalian cardiac progenitors. Proc Natl Acad Sci USA 104:10894-10899 
21. Leung JY, Kolligs FT, Wu R, Zhai Y, Kuick R, Hanash S et al (2002) Activation of AXIN2 expression by beta-catenin-T cell factor. A feedback repressor pathway regulating Wnt signaling. J Biol Chem 277:21657-21665

22. Lin L, Cui L, Zhou W, Dufort D, Zhang X, Cai CL et al (2007) Beta-catenin directly regulates Islet 1 expression in cardiovascular progenitors and is required for multiple aspects of cardiogenesis. Proc Natl Acad Sci USA 104:9313-9318

23. Lloyd-Jones D, Adams R, Carnethon M, De SG, Ferguson TB, Flegal K et al (2009) Heart disease and stroke statistics-2009 update: a report from the American Heart Association Statistics Committee and Stroke Statistics Subcommittee. Circulation 119:480-486

24. Lustig B, Jerchow B, Sachs M, Weiler S, Pietsch T, Karsten U et al (2002) Negative feedback loop of Wnt signaling through upregulation of conductin/axin2 in colorectal and liver tumors. Mol Cell Biol 22:1184-1193

25. Maejima Y, Adachi S, Ito H, Hirao K, Isobe M (2008) Induction of premature senescence in cardiomyocytes by doxorubicin as a novel mechanism of myocardial damage. Aging cell 7:125136

26. Masckauchan TN, Agalliu D, Vorontchikhina M, Ahn A, Parmalee NL, Li CM et al (2006) Wnt5a signaling induces proliferation and survival of endothelial cells in vitro and expression of MMP-1 and Tie-2. Mol Biol Cell 17:5163-5172

27. Mirotsou M, Zhang Z, Deb A, Zhang L, Gnecchi M, Noiseux N et al (2007) Secreted frizzled related protein 2 (Sfrp2) is the key Akt-mesenchymal stem cell-released paracrine factor mediating myocardial survival and repair. Proc Natl Acad Sci USA 104:1643-1648

28. Miura T, Miki T (2008) Limitation of myocardial infarct size in the clinical setting: current status and challenges in translating animal experiments into clinical therapy. Basic Res Cardiol 103:501-513

29. Oh H, Bradfute SB, Gallardo TD, Nakamura T, Gaussin V, Mishina $Y$ et al (2003) Cardiac progenitor cells from adult myocardium: homing, differentiation, and fusion after infarction. Proc Natl Acad Sci USA 100:12313-12318

30. Oka T, Xu J, Molkentin JD (2007) Re-employment of developmental transcription factors in adult heart disease. Semin Cell Dev Biol 18:117-131

31. Polesskaya A, Seale P, Rudnicki MA (2003) Wnt signaling induces the myogenic specification of resident CD45+ adult stem cells during muscle regeneration. Cell 113:841-852
32. Qu J, Zhou J, Yi XP, Dong B, Zheng H, Miller LM et al (2007) Cardiac-specific haploinsufficiency of beta-catenin attenuates cardiac hypertrophy but enhances fetal gene expression in response to aortic constriction. J Mol Cell Cardiol 43:319-326

33. Qyang Y, Martin-Puig S, Chiravuri M, Chen S, Xu H, Bu L et al (2007) The renewal and differentiation of Isl1+ cardiovascular progenitors are controlled by a Wnt/beta-catenin pathway. Cell Stem Cell 1:165-179

34. Reya T, Clevers H (2005) Wnt signalling in stem cells and cancer. Nature 434:843-850

35. Samarzija I, Sini P, Schlange T, Macdonald G, Hynes NE (2009) Wnt3a regulates proliferation and migration of HUVEC via canonical and non-canonical Wnt signaling pathways. Biochem Biophys Res Commun 386:449-454

36. Tiede K, Melchior-Becker A, Fischer JW (2010) Transcriptional and posttranscriptional regulators of biglycan in cardiac fibroblasts. Basic Res Cardiol 105:99-108

37. Timmers L, Sluijter JP, van Keulen JK, Hoefer IE, Nederhoff MG, Goumans MJ et al (2008) Toll-like receptor 4 mediates maladaptive left ventricular remodeling and impairs cardiac function after myocardial infarction. Circ Res 102:257-264

38. van de Schans V, van den Borne SW, Strzelecka AE, Janssen BJ, van der Velden JL, Langen RC et al (2007) Interruption of Wnt signaling attenuates the onset of pressure overload-induced cardiac hypertrophy. Hypertension 49:473-480

39. van Laake LW, Passier R, Monshouwer-Kloots J, Nederhoff MG, Ward-van OD, Field LJ et al (2007) Monitoring of cell therapy and assessment of cardiac function using magnetic resonance imaging in a mouse model of myocardial infarction. Nat Protoc 2:2551-2567

40. Wang X, Xiao Y, Mou Y, Zhao Y, Blankesteijn WM, Hall JL (2002) A role for the beta-catenin/T-cell factor signaling cascade in vascular remodeling. Circ Res 90:340-347

41. Zamora M, Manner J, Ruiz-Lozano P (2007) Epicardium-derived progenitor cells require beta-catenin for coronary artery formation. Proc Natl Acad Sci USA 104:18109-18114

42. Zelarayan LC, Noack C, Sekkali B, Kmecova J, Gehrke C, Renger A et al (2008) Beta-Catenin downregulation attenuates ischemic cardiac remodeling through enhanced resident precursor cell differentiation. Proc Natl Acad Sci USA 105:19762-19767

43. Zhang Z, Deb A, Zhang Z, Pachori A, He W, Guo J et al (2009) Secreted frizzled related protein 2 protects cells from apoptosis by blocking the effect of canonical Wnt3a. J Mol Cell Cardiol 46:370-377 